\title{
Reported recovered memories of child sexual abuse
}

\section{Recommendations for good practice and implications for training, continuing professional development and research}

The College recognises the severity and significance of child sexual abuse and the suffering experienced both at the time of the abuse and in adult life.

The difference between incestuous fathers and paedophiles is less distinct than was previously thought. Those who sexually abuse children share many characteristics including verbal denial even in the face of clear evidence, recidivism, secrecy, minimisation, rationalisation and justification of their actions among others. These are often maintained even after criminal conviction.

Nevertheless, the growth of litigation against alleged perpetrators and therapists and the risk of bringing the profession into disrepute makes it necessary to alert psychiatrists to the possibility of 'false memories'. In this context a 'recovered memory' is one in which traumatic events have been totally forgotten until 'released' or recovered in therapy or as a result of some other trigger or experience. A 'false memory' is one which is not based on events which have occurred.

Memories are constantly forgotten and recovered, but we are here concerned with the alleged forgetting and recovery of memories of prolonged and repeated child sexual abuse, typically from childhood into adolescence. Concern about recovered memories which have no factual basis should be concentrated on those cases where patients report having had no memory whatsoever of abuse which continued over many years. In the United States concern about such recovered memories led to the use of the term False Memory Syndrome which, though misleading, has now gained wide usage.

Memory is a complex field of study which has generated an enormous literature and a plethora of theories. The evidence shows that memories of events which did not in fact occur may develop and be held with total conviction. Such memories commonly develop under the influence of individuals or situations which encourage the development of strong beliefs. They have often been described as arising within therapy, sometimes involving psychiatrists or other mental health workers, as well as psychotherapists.
Although the following recommendations are particularly concerned with the use of specific memory recovery techniques, it is important to emphasise that distortion of memory may occur in any therapeutic situation. Psychiatrists need to be aware of the techniques employed by other members of their team, including semi-autonomous practitioners. Any professionals, including senior psychiatrists, working with cases of sexual abuse or recovered memories should have access to expert advice and the opportunity for regular peer supervision.

\section{Recommendations for good practice}

(a) The welfare of the patient is the first concern of the psychiatrist. Concern for the needs of family members and others may also be necessary, within the constraints imposed by the need for confidentiality.

(b) In children and adolescents, symptoms and behaviour patterns may alert the clinician to the possibility of current sexual abuse, but these are no more than indicators for suspicion. Previous sexual abuse in the absence of memories of these events cannot be diagnosed through a checklist of symptoms.

(c) Psychiatrists are advised to avoid engaging in any 'memory recovery techniques' which are based upon the expectation of past sexual abuse of which the patient has no memory. Such 'memory recovery techniques' may include drug-mediated interviews, hypnosis, regression therapies, guided imagery, 'body memories'. literal dream interpretation and journaling. There is no evidence that the use of consciousness-altering techniques, such as drug-mediated interviews or hypnosis can reveal or accurately elaborate factual information about any past experiences including childhood sexual abuse. Techniques of regression therapy including age regression' and hypnotic regression are of unproven effectiveness.

(d) Forceful or persuasive interviewing techniques are not acceptable in psychiatric practice. Doctors should be aware that patients are 
susceptible to subtle suggestions and reinforcements whether these communications are intended or unintended.

(e) The psychiatrist should normally explore his or her doubts with the patient about the accuracy of recovered memories of previously totally forgotten sexual abuse. This may be particularly important if the patient intends to take action outside the therapeutic situation. Memories, however emotionally intense and significant to the individual, do not necessarily reflect factual events.

(f) Adult patients reporting previously forgotten abuse may wish to confront the alleged abuser. Such action should not be mandated by the psychiatrist and likewise it is rarely appropriate to discourage or even to forbid the patient from having contact with the alleged abuser or family members. The psychiatrist should help the patient to think through the possible consequences of confrontation with the alleged abuser. In these circumstances it is appropriate to encourage the search for corroboration.

(g) Psychiatrists should resist vigorously any move towards the compulsory reporting of all allegations or suspicions by adults of sexual abuse during childhood. Mandatory reporting is entirely appropriate where children or adolescents spontaneously report current or recent abuse. Hints at the possibility or suspicion of current sexual abuse always need to be carefully evaluated and investigated.

(h) It may be legitimate not to question the validity of a recovered memory while it remains within the privacy of the consulting room, though there is a risk in colluding with, and creating, a life history based upon a false belief. Action taken outside the consulting room, including revealing the accusations to any third party, must depend upon circumstances and upon the wishes of the patient.

(i) Once the accusation is taken outside the consulting room, especially if any question of confrontation or public accusation arises, there can rarely be any justification for refusal to allow a member of the therapeutic team to meet family members.

(ii) Where the alleged abuser is still in touch with children at risk serious consideration must be given to informing the appropriate social service department. This must be done if there are reasonable grounds for believing that the alleged assault or assaults actually took place and that other children may now be at risk. If the case is reported by others the psychiatrist should also be prepared to state clearly if he or she believes that the grounds for the accusation are inadequate or unreasonable. (iii) The patient may wish to seek legal advice, possibly with a view to the prosecution of or litigation against the alleged abuser. It is unwise to encourage or discourage legal action and inappropriate to make any decision about this a condition of continuing treatment. The patient should always be encouraged to consider the possible consequences of such action.

(i) Although there are doubts about the validity of diagnoses of dissociative identity disorder (formerly multiple personality disorder) it is asserted by some that this condition is frequently associated with a history of childhood sexual abuse. There seems little doubt that some cases of multiple personality are iatrogenically determined and psychiatrists should be careful to ensure that they do not directly encourage patients to develop 'alters' in whom they may invest aspects of their personality, fantasies or current problems. Any spontaneous presentation of dissociative identity disorder should be sympathetically considered but should not be made the subject of undue attention, nor should the patient be encouraged to develop further 'multiples'. Psychiatrists should be particularly aware of the unreliability of memories reported in these cases. Since there is no settled view on the validity of these diagnostic concepts there is a case for the preparation of a consensus statement which would need to be based upon a substantial literature review.

\section{Implications for training and research}

\section{Postgraduate training}

Postgraduate psychiatric trainees should have:

- specific and detailed instruction in the psychological and neurobiological foundations of memory, including a critique of historical and current theories.

- a good understanding of the clinical and epidemiological aspects of child sexual abuse and child protection procedures. They should know of the associations between childhood sexual abuse and adult psychopathology and their empirical validity.

- instruction on the dangers of suggestion and suggestibility, and a keen awareness that opinions and prejudices in the therapist may have a profound effect upon their patients. They should also understand how to minimise possible adverse effects of such influences.

- an awareness of the need to obtain collateral histories to amplify the clinical history and examination. 
- an awareness of the need to review their beliefs and practices in the light of new evidence and a recognition of the limitations of their own knowledge and expertise.

\section{Continuing professional development}

Psychiatrists may not have made themselves aware of the developments in the understanding of memory, suggestion or of child sexual abuse and its possible consequences for adult psychotherapy. Continuing professional development should therefore ensure that, through courses and authoritative reviews, psychiatrists are kept up to date on these topics.

It is appropriate that all psychiatrists should have a general understanding of child abuse and its consequences. The skills required for the assessment or therapy of the abused and their families require additional training and experience. All psychiatrists should be open to new knowledge and ready to modify their beliefs and practices accordingly. Psychiatry as a profession should know the limits of its knowledge and experience.

\section{Research}

Further research is required into the nature and validity of such concepts as repression, dissociation and the psycho-neuro-physiology of traumatic memories of all kinds. More precise definition of child sexual abuse, accurate recording of its type and duration, of the relationship between the victim and the perpetrator and the age of commencement and duration of the abuse are required for further studies. These studies need to examine the relationship between different varieties and severities of child sexual abuse and later adult psychopathology and to consider the influence of early experience in general, including the effects of physical and emotional abuse.

Royal COLlege of PSychiatrists' WORKING GROUP ON REPORTED RECOVERED MEMORIES OF CHILD Sexual ABuSe: Professor Sydney Brandon (Chair), Dr Janet Boakes, Dr Danya Glaser, Professor Richard Green. Dr James MacKeith and Dr Peter Whewell

Approved by Council, 24 June 1997 